\section{SA1-01 タンパク質共進化と分子進化速度の共変動}

Co-evolution of proteins and covariation of evolutionary rate

Hirohisa Kishino (1) ((1) University of Tokyo)

The rate of molecular evolution is the product of the effective population size, mutation rate, and the fixation probability of the new alleles. When most of new alleles are either neutral or deleterious, the rate of molecular evolution is the product of the mutation rate and the extent of the functional constraint. While the mutation rate co-varies among loci in the genome, the different proteins are generally under different functional constraint. Therefore, the variation of the evolutionary rate due to the change in functional constraint may be extracted by removing the overall variation of the rate of genome evolution. The removal of background rate variation is particularly important in the analysis of co-evolution. By comparing protein sequences, it is possible to estimate the amount of evolutionary events among them, which are described as the branch lengths of the phylogenetic tree. Bayesian procedures with smoothness priors for the variation of evolutionary rate estimate the divergence times and the variable evolutionary rates. To sensitivity and selectivity of the detection of co-evolution depends on the robustness and the power of the Bayesian analysis.

\section{SA1-02 バルナーゼとバルスターの相互作用の共進化解析}

Co-evolutionary analyses of interactions between barnase and barstar

Teikichi Ikura (1), Yoshiaki Urakubo (1), Nobutoshi Ito (1) ((1) Tokyo Medical and Dental University)

Barnase, an extracellular ribonuclease of Bacillus amyloliquefaciens, forms very tight complex with its intracellular inhibitor barstar: the association constant is more than $5 \times 109 \mathrm{~s}^{-1} \bullet \mathrm{M}^{-1}$ and the dissociation constant is $1.3 \times 10^{-14} \mathrm{M}$, which corresponds to $19.0 \mathrm{kcal} \bullet \mathrm{mol}^{-1}$ of the binding free energy. Such tight interaction between them must result from the toxic nature of barnase, which is lethal to the cells when expressed without its inhibitor. As shown in some reports, the cells expressing mutant barnase with low RNase activity do not require high activity of the inhibitor to survive. Evolution of the activity of barnase has presumably coevolved the inhibitory activity of barstar, resulting in their extraordinarily tight interaction. Therefore, the interaction between barnase and barstar is an excellent system to investigate co-evolution in the protein-protein interaction.

We have investigated and characterized the interaction between barnase and barstar by protein engineering techniques combined with surface plasmon resonance measurement, X-ray crystallography, and molecular dynamics simulation. Furthermore, we have elucidated that the water molecules penetrating into the binding interface drastically affect the interaction between them.

At this symposium, we will discuss various elements for maintaining and improving the stability and specificity of the interaction between them from the viewpoint of co-evolution.

\section{SA1-03 共進化情報によるタンパク質間相互作用予測}

Prediction of protein-protein interaction with co-evolutionary information

Hiroyuki Toh (1), Tetsuya Sato (1) ((1) Medical Institute of Bioregulation, Kyushu University)

Interacting proteins are considered to evolve together through the interaction, which is called co-evolution. Many methods have been developed to predict protein-protein interaction by using the co-evolutionary information at the molecular level. Mirror tree method is one of such approaches, where the phylogenetic trees of the interacting proteins are assumed to resemble due to the co-evolution. In the actual operation, the distance matrices are compared, instead of the phylogenetic trees. The similarity between distance matrices is evaluated with Pearson's correlation coefficient. If the correlation coefficient is close to one, the corresponding proteins are predicted to interact. Because of the simplicity, the method has been widely used as a in silico method to analyze protein-protein interaction, and many variations of the method have been developed. At the same time, the problems of the mirror tree method have been revealed. One of the problems is the high false positive ratio of the mirror tree prediction. We considered that the false positive is caused by the evolutionary information of source organisms included in the distance matrices. Based on this assumption, we have developed several methods to treat this problem. In the methods, the evolutionary information of source organisms is removed from the distance matrices, and the residuals are compared. In this talk, we will discuss the performance of our methods, together with the problems of mirror tree method.

\section{SA1-04 BPTI 変異体の熱安定性とタンパク質間相互作用の共進化的視点 からの解析}

Analysis of thermal stability and protein-protein interaction in BPTI from a co-evolutionary viewpoint

Yutaka Kuroda (1) ((1) Tokyo University of Agriculture and Technology (TUAT))
It is well established that the structure and stability of small globular proteins, which in turn shapes their functions, are determined by their amino acid sequences. A protein amino acid sequence can be viewed as being controlled by two conceptually distinct factors. First, physico-chemical factors select a large pool of amino acid sequences that fold into native structures, which can all potentially perform the intended function. Second, evolution selects some or many sequences from the above pool, so that the structure can efficiently and/or adequately exert its biological activity in its biological context. When activity is mediated by protein-protein (or peptide) interaction, co-evolution between the interacting proteins may play an important role in the second stage of the sequence selection. Here, we aim at analyzing the relative importance of the two factors in determining the sequence and thus the fold and stability of our model protein BPTI( Bovine Pancreatic Trypsin Inhibitors). To this end, we discuss the results of a multiple alanine scanning experiment based on a co-evolutionary analysis of the trypsin/BPTI interaction. We will also examine whether or how co-evolutionary analysis could provide an engineering tool for enhancing protein stability.

\section{SA1-05 タンパク質共進化機構の集団遺伝学的考察}

Population gentical study on the mechanism of co-evolution of proteins

Masaru Iizuka (1) ((1) Kyushu Dental College)

Compensatory mutations are individually deleterious but harmless in appropriate combinations. Many experimental results and those based on phylogeneticcomparative analysis are reported that suggest molecular evolution by compensatory mutations. Compensatory mutations are recognized as correlated mutations or coevolution in protein science. A mutation on one residue where the functional constraints are operating can be compensated by an additional mutation of a complementary residue across the interface. These correlated mutations enable the coevolution of two proteins that can lead to high specificity and high affinity. Kimura (J. Genetics 64 (1985) 7-19) investigated population genetic mechanism of molecular evolution by compensatory mutations. This is a model (compensatory neutral mutation model) for haploid organisms. We extend this model to diploid organisms. If deleterious effects of single mutants are recessive selection against the intermediate deleterious states of single mutants will be less effective for diploid organisms. In addition to the effects of dominance in selection, there exists a difference between monomer formation and heterodimer formation of gene products for diploid organisms. Considering that dominance effects of selection and heterodimer formation of gene products may affect the rate of compensatory evolution, we investigate compensatory neutral mutation models for diploid organisms. Our theoretical analysis shows that these factors play an important role in reducing the evolutionary rate of compensatory mutations (Ichinose et al. Theor. Popul. Biol. 74 (2008) 199-207)

1SA2-02 Intracellular localization of abnormal isoform of prion protein Motohiro Horiuchi (1) ((1) Graduate School of Veterinary Medicine, Hokkaido Univeristy)

The propagation of prion in neurons is believed to be tightly associated with neuronal degeneration. An elucidation of the intracellular localization of PrPSc will facilitate the understanding of molecular mechanism of prion propagation. Recently we found mAb 132 recognizing the region adjacent to the most amyloidgenic region of $\operatorname{PrP}$ (aa112-119) is useful for detection of PrPSc in IFA. Extensive analysis of the intracellular localization of PrPSc by a double staining with mAb 132 and organella markers revealed the co-localization of PrPSc with lysosome markers as reported previously. The most prominent finding, however, was the presence of PrPSc at juxtanuclear region that was not co-localized with lysosome markers. PrPSc detected at juxtanuclear region existed very close to trans-Golgi network (TGN), and appeared to be closely associated with an intracellular transport pathway of Siga toxin beta subunit. A low-temperature incubation that blocks the retrograde transport from endosome to TGN affected the PrPSc accumulation at juxtanuclear region. Furthermore, PrPSc appears to be co-localized at least some part with vacuolar protein sorting (Vps) 35, Rab 9, or Clint1, which are involved in the machineries associated with retrograde-transport of proteins from endosome to TGN. The amount of PrPSc decreased and the juxtanuclear accumulation of PrPSc disappeared when the expressions of some of those molecules were down-regulated by small interfering RNA. These results suggest that the early/recycling endosome and/or the retrograde-transport pathway of proteins from endosome to TGN may be involved in the synthesis of PrPSc.

1SA2-03 Investigating the role of exosomes in the processing of proteins associated with prion and Alzheimer's diseases

Andrew F. Hill (1) ((1) University of Melbourne, Department of Biochemistry and Molecular Biology)

Exosomes are small membranous vesicles secreted by a number of cell types including neurons and can be isolated from conditioned cell media or bodily fluids such as urine and plasma. Exosome biogenesis involves the inward budding of endosomes to form multivesicular bodies (MVB). When fused with the plasma membrane, the MVB releases the vesicles into the extracellular environment as 